\title{
The Influence of microRNA on Signaling Pathways Activity in Radiosensitive and Radioresistant Cancer Cell Lines after Radiation Exposure
}

\author{
DOI: $10.17691 / \mathrm{stm} 2017.9 .2 .03$
}

Received June 8, 2016

D.A. Maslennikova, Research Engineer, Laboratory of Molecular and Cellular Biology, S.R. Kapitsa Technological Research Institute;

A.V. Khokhlova, Junior Researcher, Laboratory of Molecular and Cellular Biology, S.P. Kapitsa Technological Research Institute;

E.S. Pogodina, Research Engineer, Laboratory of Molecular and Cellular Biology, S.P. Kapitsa Technological Research Institute;

D.A. Zazhoma, Undergraduate Student;

S.N. Vorsina, Undergraduate Student;

E.A. Beloborodov, Graduate Student;

V.V. Saenko, PhD, Senior Researcher, Laboratory of Molecular and Cellular Biology, S.P. Kapitsa

Technological Research Institute;

Y.V. Saenko, DSc, Head of Laboratory of Molecular and Cellular Biology, S.P. Kapitsa Technological Research Institute

Ulyanovsk State University, 42 Leo Tolstoy St., Ulyanovsk, 432017, Russian Federation

The aim of the investigation was to study microRNA expression and its influence on signaling pathways activity in radioresistant and radiosensitive cell lines exposed to radiation.

Materials and Methods. Radioresistant K562 cell line and radiosensitive HL-60 and Raji cell lines were used in the study. Cell survival was estimated after exposure to $4 \mathrm{~Gy}$ gamma radiation. MicroRNA composition was studied 1, 4 and $24 \mathrm{~h}$ after radiation exposure using massively parallel sequencing method. Bioinformatics analysis was performed using GenXpro and PANTHER database.

Results. After single $4 \mathrm{~Gy}$ radiation exposure, the number of cells with the signs of necrosis increased several times in radiosensitive cell lines as compared to control samples. MicroRNA hsa-miR-590-3p was found in each studied cell line at every stage of the experiment. The most significant differences between radioresistant and radiosensitive cell lines were observed in dynamics of microRNA influence on Integrin signaling pathway and General transcription by RNA polymerase I pathway.

Conclusion. MicroRNA hsa-miR-590-3p dynamics and expression level were found to correlate with cancer cell radiosensitivity. Its influence on radioresistant and radiosensitive cell lines was different: in radioresistant K562 cell line, the inhibitory effect of microRNA on Integrin signaling pathway was reduced and this effect on General transcription by RNA polymerase I pathway was increased.

Key words: microRNA; hsa-miR-590-3p; gene expression; cancer cell radiosensitivity; gamma radiation; Integrin signaling pathway; General transcription by RNA polymerase I.

Radioresistance of malignant tumors is a serious problem for radiotherapy and the main barrier to achieving the maximum efficacy of radiation therapy in cancer treatment.

Radioresistance of cancer cells is a property based on a number of genetic mutations and normal gene expression impairment, which, in turn, alters cancer cell sensitivity to radiotherapy. Gene expression impairment leads to abnormalities in cell metabolism and changes in regulation of cell processes, particularly, those associated with programmed cell death. The phenomenon of radioresistance is formed at the supramolecular level and associated with the capability of cancer cells to abort launching the mechanisms of programmed cell death induced by radiation exposure
$[1,2]$. The mechanisms of programmed cell death, apoptosis and autophagy, are launched in normal cells when cell damage repair, especially, DNA damage, is impossible. Programmed cell death induced in stress exposure is not only associated with the extent of DNA damage, but involves a great number of other signaling mechanisms and depends on their interaction [3, 4]. The functioning of signaling and metabolic mechanisms is associated with gene expression regulation at the transcriptional and post-transcriptional levels.

Regulation of messenger RNA (mRNA) degradation by means of microRNA is the main mechanism of gene expression regulation at the post-transcriptional level. MicroRNA is a small (20-25 pairs of nucleotides) non-coding RNA affecting transcriptional and post-

For contacts: Yuriy V. Saenko, e-mail: saenkoyv@yandex.ru 
transcriptional gene regulation by silencing the expression [5, 6]. More than a thousand of microRNAs have been found in human genome, each of them being capable of regulating hundreds of mRNAs, therefore, they play a critical role in many cellular processes, including radiosensitivity and radioresistance of cells. MicroRNA was found to regulate expression of nearly $60 \%$ of all protein-coding genes [7].

Recent investigations show that microRNAs play an important role in cancer pathogenesis [8, 9]. Their expression changes under the influence of ionizing radiation, which suggests their participation in cellular response to ionizing radiation [10]. The conducted studies [11-14] have demonstrated the relation between certain microRNAs and cancer cell radioresistance, which has provided the possibility to reveal several dozens of microRNA differentially expressed in radioresistant and radiosensitive cell lines. However, microRNAs typical of all radioresistant cell lines have not been found. This is explained by the fact that gene expression regulation by means of microRNA is a complex process, in which a single microRNA can regulate expression of many genes, and expression of a single gene can be regulated by many different microRNAs. In this case, a more promising approach to estimating the role of microRNA is exploring its influence on signaling pathways.

The aim of the investigation was to study microRNA expression in radioresistant and radiosensitive cell lines after radiation exposure and to analyze its impact on signaling pathways activity.

Materials and Methods. The experiment was carried out on HL-60 cell line (human promyelocytic leukemia), K562 (radioresistant suspension line of human chronic myeloid leukemia), Raji (human lymphoblastoid cell line).

Cells were cultured at $37^{\circ} \mathrm{C}$ under $5 \% \quad \mathrm{CO}_{2}$ atmosphere of $98 \%$ humidity using RPMI-1640 medium containing L-glutamine with $10 \%$ fetal bovine serum and $50 \mu \mathrm{g} / \mathrm{ml}$ gentamicin.

The cell lines were exposed to gamma radiation generated by linear accelerator Elekta Synergy (Elekta, Sweden) at a room temperature at a dose of $4 \mathrm{~Gy}$ (photon energy $10 \mathrm{MeV}$ ) in the logarithmic phase of growth. Exposure time amounted to $55 \mathrm{~s}$ at $21-22^{\circ} \mathrm{C}$. Irradiated field was $16 \times 20 \mathrm{~mm}$. The cells were placed in $5 \cdot 10^{5} / \mathrm{ml}$ of medium in 6 -well plates of $2.5 \mathrm{ml}$ well volume.

Control groups were not subjected to any exposure.

Cell survival was estimated by cell staining with a mixture of fluorescent dyes acridine orange and propidium iodide. The stained cells were analyzed using fluorescence microscope. The cells with the signs of necrosis were stained with orange, live cells - with green [15].

Total RNA enriched with microRNA was isolated from the cells 1,4 and $24 \mathrm{~h}$ after the exposure using Absolutely RNA miRNA Kit (Agilent Technologies, USA). The quality of isolated RNA was assessed by the ratio of 18S/28S RNA using capillary electrophoresis device Agilent Bioanalyzer 2100 (Agilent Technologies, USA). Samples with integrity number $\mathrm{RIN}>8.0$ were selected for further work. NEBNext Small RNA Library Prep Set (NEB, UK) was used to prepare the library of complementary DNA (microRNA cDNA) and to perform adapter ligation and bar coding. MicroRNA cDNA library cleaning was performed by means of electrophoresis with $6 \%$ polyacrylamide gel. cDNA fragments between 145 and $160 \mathrm{bp}$ corresponding to microRNA were excised from the gel, eluted and precipitated by $80 \%$ ethanol. cDNA pellet was air-dried and re-suspended in $10 \mu \mathrm{L}$ of water processed with diethyl pyrocarbonate. The quantity of cDNA in each final microRNA library was measured using Qubit fluorometer (Invitrogen, USA). Equimolar amounts (2 $\mathrm{nM}$ ) of each library were pooled and sequenced by means of Illumina MiSeq system (Illumina, USA) using a $150 \mathrm{bp}$ kit for single-end read.

FASTQ files for each cell line were produced as a result of sequencing. Their bioinformatics analysis was performed on GenXPro omiRas platform (Germany) [16], the obtained data were arranged in tables containing the quantity of microRNA denominations and microRNA number of readings normalized by $10^{5}$ reads.

Search for genes whose expression is regulated by microRNA was carried out using GenXPro omiRas platform (Germany) [16]. MicroRNAs with normalized expression were selected for further study ( $>100$ pieces per sample). As a result, there was compiled a list of genes whose expression is regulated by microRNA for each cell line and experimental point.

Search for signaling pathways involving these genes was carried out using PANTHER database (USA) (http://www.pantherdb.org/). Since each microRNA is equally likely to take part in each signaling pathway, the normalized quantity of transcripts from each microRNA was divided by the number of signaling pathways. The obtained values of normalized microRNA expression for each signaling pathway were then summed up to obtain the sum of normalized expressions (SNE) of all microRNAs participating in post-transcriptional expression regulation of genes involved in that signaling pathway. Test and control sums of normalized expressions were compared and presented as log2 FC (a binary logarithm of the ratio of test and control SNE). Graphs were plotted relying on the obtained data. Those signaling pathways whose log2 FC SNE values were more than 0.5 or less than -0.5 at least in one experiment (log2 $\mathrm{FC}>0.5$ and $\log 2 \mathrm{FC}<-0.5)$ were selected for plotting.

Results. Figure 1 shows the number of cells with the signs of necrosis in control and after exposure to $4 \mathrm{~Gy}$ ionizing radiation in the three cell lines: K562, HL-60, Raji. In K562 cell line, the number of cells with the signs of necrosis in control equals $7.23 \%$, it amounts to $13.56 \%$ after a single exposure to $4 \mathrm{~Gy}$ radiation. In radiosensitive $\mathrm{HL}-60$ cell line, the number of cells with the signs of necrosis increases several times after a 
Figure 1. A proportion of cells with the signs of necrosis in control and after 4 Gy radiation exposure in K562, HL-60, Raji cell lines

* Statistically significant difference of values compared to control; \# compared to K562 cell line; $p<0.05$
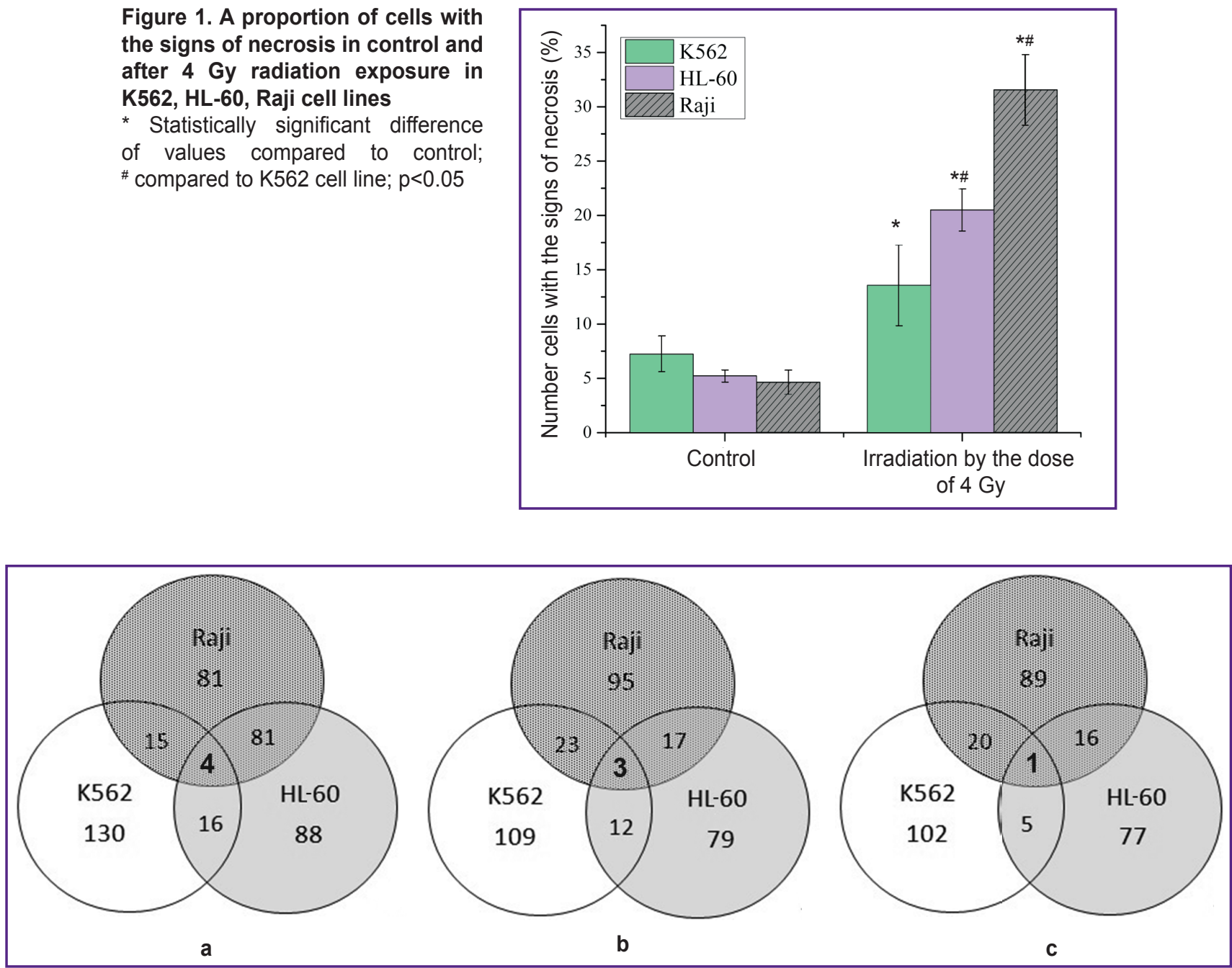

Figure 2. The number of differentially expressed microRNAs in K562, HL-60, Raji cell lines: (a) $1 \mathrm{~h}$ after radiation exposure; (b) $4 \mathrm{~h}$ after radiation exposure; (c) $24 \mathrm{~h}$ after radiation exposure

single 4 Gy radiation exposure, as compared to control, and amounts to $20.5 \%$ instead of $5.23 \%$. In Raji cell line, the proportion of cells with the signs of necrosis after radiation exposure equals $31.56 \%$.

As a result of microRNA sequencing and subsequent analysis, differentially expressed microRNAs were revealed in each cell line 1, 4 and $24 \mathrm{~h}$ after irradiation with $4 \mathrm{~Gy}$ of X-ray. Venn diagram (Figure 2) demonstrates the intersection of microRNA quantities of all cell lines. One hour after radiation exposure there were found 4 differentially expressed microRNAs characteristic of all three cell lines under study - hsa-miR-5701, hsa-miR-590-3p, hsa-miR-5010-3p, hsa-miR-3607-5p (Figure 2 (a)). After $4 \mathrm{~h}$, only hsa-miR-590-3p, hsa-miR5010-3p, hsa-miR-3607-5p appeared to be present at the intersection of quantities and after $24 \mathrm{~h}$ there was only one microRNA: hsa-miR-590-3p (Figure 2 (b), (c)).

Post-transcriptional regulation of gene expression by microRNA has a number of specific features, one of them being the capability to regulate expression of many genes by a single microRNA and expression of a single gene by multiple microRNAs. Therefore, investigating only differential microRNA expression is not sufficient for understanding the differences between radioresistant and radiosensitive cell lines. To gain a deeper insight into the reasons for cancer cell radioresistance and the role of microRNA in this process, we have performed bioinformatics analysis of interaction between microRNA and intracellular signaling and metabolic pathways (Figure 3). The graphs depict microRNA exerting "pressure" on the activity of certain signaling pathways. The value pressure is presented as log2 FC SNE. Negative log2 FC SNE values indicate that microRNA pressure on the signaling pathway increases, positive values imply pressure decrease.

Figure 3 demonstrates that $\mathrm{K} 562$ cell line is characterized by unstable dynamics of microRNA influence on signaling pathways activity. This instability shows itself in variation of log2 FC SNE values from negative to positive ones (Figure 3 (b)). In K562 


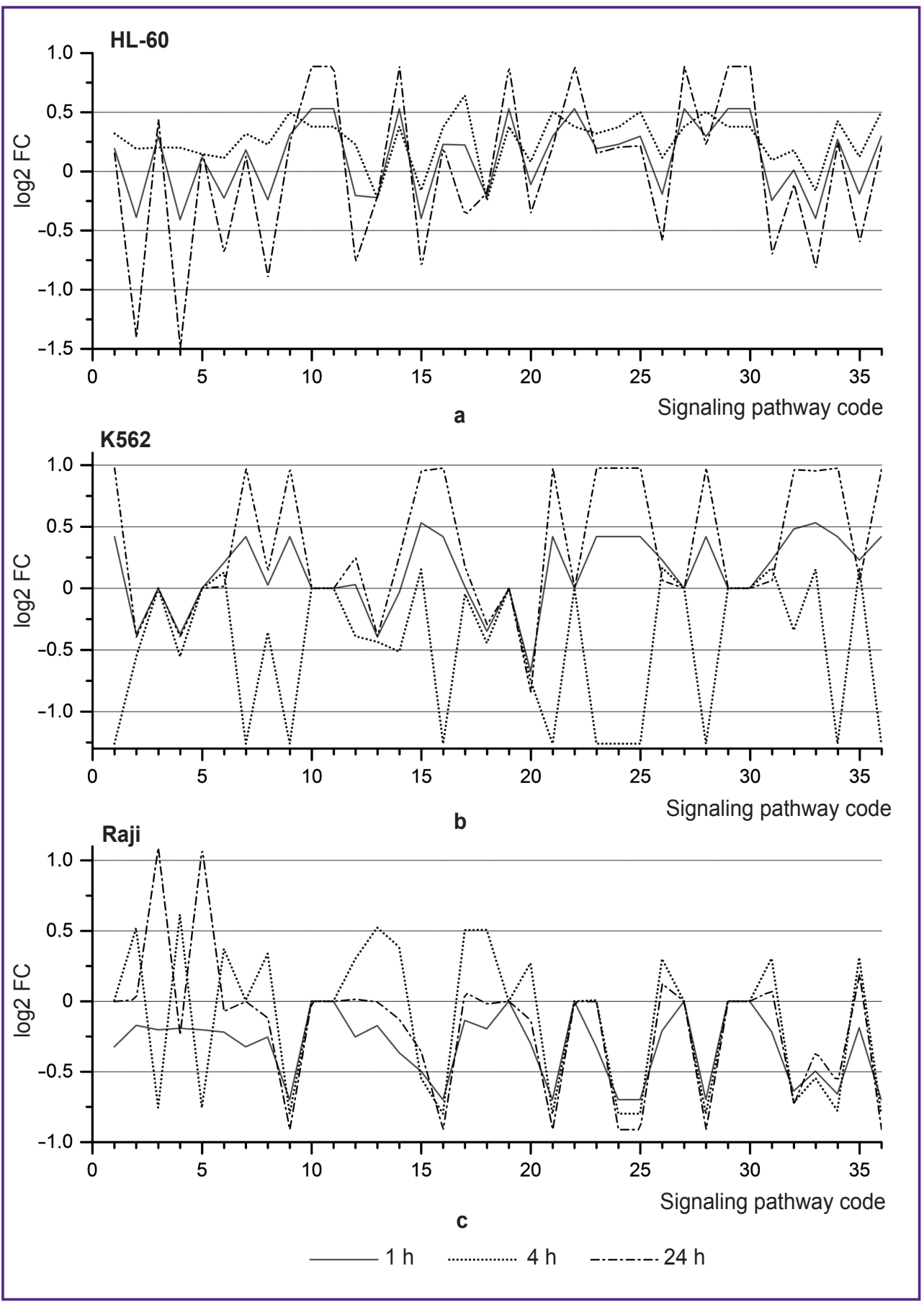

Figure 3. Dynamics of microRNA influence on signaling pathways activity 1, 4, $24 \mathrm{~h}$ after radiation exposure in HL-60 cancer cell line (a); K562 cancer cell line (b); Raji cancer cell line (c)

Signaling pathway code: Adrenaline and noradrenaline biosynthesis (1); Angiotensin II-stimulated signaling through $G$ proteins and beta-arrestin (2); Axon guidance mediated by netrin (3); Axon guidance mediated by semaphorins (4); Axon guidance mediated by Slit/Robo (5); B cell activation (6); Blood coagulation (7); Cadherin signaling pathway (8); de novo purine biosynthesis (9); de novo pyrimidine ribonucleotides (10); Endogenous cannabinoid signaling (11); Endothelin signaling pathway (12); Enkephalin release (13); GABA-B receptor II signaling (14); General transcription by RNA polymerase I (15); General transcription regulation (16); Hedgehog signaling pathway (17); Heterotrimeric G-protein signaling pathway-Gi alpha and Gs alpha mediated pathway (18); Heterotrimeric G-protein signaling pathway-rod outer segment phototransduction (19); Integrin signaling pathways (20); N-acetylglucosamine metabolism (21); Nicotine pharmacodynamics pathway (22); Nicotinic acetylcholine receptor signaling pathway (23); O-antigen biosynthesis (24); Pyridoxal-5-phosphate biosynthesis (25); Ras pathway (26); Salvage pyrimidine ribonucleotides (27); Serine/glycine biosynthesis (28); Sulfate assimilation (29); Synaptic vesicle trafficking (30); T cell activation (31); Transcription regulation by bZIP transcription factor (32); Tryptophan biosynthesis (33); Ubiquitin proteasome pathway (34); VEGF signaling pathway (35); Vitamin B6 metabolism (36). log2 FC — binary logarithm 
cell line, 11 signaling pathways are subject to such variation, in Raji cell line -3 values (Figure 3 (c)), though we have found no such signaling pathways in HL-60 cell line (Figure $3(a)$ ). The most significant differences between radioresistant K562 cell line and radiosensitive HL-60 and Raji cell lines are observed in dynamics of microRNA pressure on Integrin signaling pathway (Figure 3, code 20) and General transcription by RNA polymerase I (Figure 3, code 15). In K562 cell line, microRNA pressure on signaling pathway General transcription by RNA polymerase I gradually declines in the course of experiment to reach the value of log2 $\mathrm{FC}=0.924 \mathrm{~h}$ after radiation exposure (See Figure $3(\mathrm{~b})$ ). In HL-60 and Raji cell lines, microRNA pressure on this signaling pathway gradually increases to reach the value of $\log 2 \mathrm{FC}=-0.4 \ldots-0.524 \mathrm{~h}$ after radiation exposure. MicroRNA pressure on Integrin signaling pathway declines in K562 cell line $1 \mathrm{~h}$ after radiation exposure ( $\log 2 \mathrm{FC}=0.51$ ), it returns to control values in $4 \mathrm{~h}$ and declines in $24 \mathrm{~h}$ to reach the value of $\log 2 \mathrm{FC}=1.01$ (See Figure 3 (b)). In HL-60 and Raji cell lines, pressure on this signaling pathway is increased actually throughout the experiment and varies in the range of $\log 2 \mathrm{FC}=-0.5$ (See Figure 3 (a), (c)).

Discussion. Three cancer cell lines of different radiosensitivity to gamma radiation have been selected for the experiment. The lowest radiosensitivity is demonstrated by K562 cell line (suspension line of human chronic myeloid leukemia), HL-60 cell line (human promyelocytic leukemia) shows moderate radiosensitivity and Raji (human lymphoblastoid cell line) has the highest radiosensitivity. Raji cell death rate after a single exposure to $4 \mathrm{~Gy}$ gamma radiation is 2 times higher than $\mathrm{K} 562$ cell death rate as compared to control (31.56 against $13.56 \%$, respectively) and $\mathrm{HL}-60$ cell death rate is higher than that of $\mathrm{K} 562$ line by $35 \%$ (See Figure 1). Thus, the cell lines used in the study have similar background, but different radiosensitivity.

MicroRNA analysis has shown that microRNAs hsamiR-590-3p are differentially expressed in all three cell lines (See Figure 2), expression being considerably weaker in $\mathrm{K} 562$ line than in HL-60 and Raji lines (4-16 cases per $10^{5}$ reads in $K 562$ versus $30-69$ cases per $10^{5}$ reads in HL-60 and Raji). MicroRNA hsa-miR-590$3 p$ inhibits mitochondrial dysfunction and oxidative stress in cells treated with 1-methyl-4-phenyl-pyridine in lines MES23.5 and SH-SY5Y [17]. The said microRNA has been proved to be closely related to many neurodegenerative disorders, particularly, it plays an important role in Parkinson disease [18-20]. Besides, JMJD1C gene has been identified as hsa-mir-590-3p target gene in the human body. Silencing the expression through hsa-miR-590-3p or JMJD1C gene knockdown increases expression of PGC-1-alpha, $C P H-1$ and TFAM genes which are the key genes regulating mitochondrial function [17]. JMJD1C gene also plays an important role in cellular response to DNA damage and is necessary for survival of acute myeloid leukemia cells [21, 22].
Radiation exposure induces increase in hsa-miR-590-3p quantity in all cell lines; however, this microRNA quantity still remains 2-3 times lower in K562 cell line than in HL-60 and Raji lines. Thus, hsa-miR-590-3p dynamics and expression intensity correlate with cancer cell radiosensitivity.

SNE analysis for all microRNAs taking part in post-transcriptional expression regulation of genes involved in certain signaling pathways makes it possible to reveal the differences in microRNA effects on signaling pathways. Bioinformatics analysis established significant differences in dynamic pattern of microRNA pressure on Integrin signaling pathway (See Figure 3, code 20) and General transcription by RNA polymerase I (See Figure 3, code 15) between radioresistant cell line $\mathrm{K} 562$ and radiosensitive cell lines HL-60 and Raji. Signal transduction via Integrin signaling pathway is closely linked to the development, progression and therapy of malignant tumors [23]. A number of papers have also reported the relationship between certain key components of Integrin signaling pathway and cancer cell radiosensitivity. For example, Integrin signaling pathway activation through beta-1integrin adhesion to extracellular matrix contributes to the increase in resistance to the development of radiation-induced genomic aberrations [24]. By contrast, inhibition of signal transduction through beta-1-integrin with the use of antibodies increases sensitivity of cancer cells to ionizing radiation [25]. Our study of microRNA influence on signaling pathways has demonstrated that Integrin signaling pathway inhibition declines after radiation exposure in radioresistant K562 cell line (Figure 3 (b), code 15). In HL-60 and Raji cell lines characterized by strong radiosensitivity, Integrin signaling pathway inhibition is present (Figure 3 (a), (c), code 15). Thus, analysis of microRNA influence on signaling pathways of radioresistant and radiosensitive cell lines has shown that inhibitory effect of microRNA on Integrin signaling pathway is reduced in radioresistant cell line.

As we have found in this study, the second signaling pathway influenced differently by microRNA in radioresistant and radiosensitive cell lines is General transcription by RNA polymerase I. RNA polymerase I in eukaryotic cells is responsible for the synthesis of ribosomal RNA (except for 5S rRNA). Regulation of ribosomal RNA synthesis is associated with protein synthesis. RNA polymerase I inhibition leads to activation of p53-dependent apoptosis [26]. Radioresistant K562 cell line with knocked out gene TP53 was used in our experiments. Launching p53-induced apoptosis in this cell line was impossible. K562 cell line genotype allows this signaling pathway to be strongly affected by microRNA without adverse effects for the cell, compared to more radiosensitive cell lines $\mathrm{HL}-60$ and Raji having TP53 gene. In our opinion, the absence of TP53 gene in K562 cell line enables it to redistribute energy more effectively in favor of repair processes. 
Conclusion. Radioresistant and radiosensitive cell lines have differences in signaling pathways regulation by microRNA. MicroRNA hsa-miR-590-3p dynamics and expression level correlate with cancer cell radiosensitivity. Analysis of microRNA influence on signaling pathways of radioresistant and radiosensitive cell lines shows that the inhibitory effect of microRNA on Integrin signaling pathway is reduced and this effect on General transcription by RNA polymerase I is increased in radioresistant $\mathrm{K} 562$ cell line.

Study Funding. The study was supported by Ministry of Education and Science of the Russian Federation within the frame of state task No.6.1617.2014/K, competition "Member of the Youth Scientific and Innovation Competition", and Russian Foundation for Basic Research No.16-04-00504.

Conflict of Interests. The authors have no conflicts of interest to disclose.

\section{References}

1. Guo L., Xiao Y., Fan M., Li J., Wang Y. Profiling global kinome signatures of the radioresistant MCF-7/C6 breast cancer cells using MRM-based targeted proteomics. J Proteome Res 2015; 14(1): 193-201, https://doi.org/10.1021/ pr500919w.

2. Kim W.-Y., Oh S.H., Woo J.-K., Hong W.K., Lee H.-Y. Targeting heat shock protein 90 overrides the resistance of lung cancer cells by blocking radiation-induced stabilization of hypoxia-inducible factor-1alpha. Cancer Res 2009; 69(4): 1624-1632, https://doi.org/10.1158/0008-5472.can-08-0505.

3. Tang Y., Cui Y., Li Z., Jiao Z., Zhang Y., He Y., Chen G., Zhou Q., Wang W., Zhou X., Luo J., Zhang S. Radiation-induced miR-208a increases the proliferation and radioresistance by targeting p21 in human lung cancer cells. $J$ Exp Clin Cancer Res 2016; 35: 7, https://doi.org/10.1186/ s13046-016-0285-3.

4. Li J.Y., Li Y.Y., Jin W., Yang Q., Shao Z.M., Tian X.S. ABT-737 reverses the acquired radioresistance of breast cancer cells by targeting Bcl-2 and Bcl-xL. J Exp Clin Cancer Res 2012; 31: 102, https://doi.org/10.1186/1756-9966-31-102.

5. Filipowicz W., Bhattacharyya S.N., Sonenberg N. Mechanisms of post-transcriptional regulation by microRNAs: are the answers in sight? Nat Rev Genet 2008; 9(2): 102-114, https://doi.org/10.1038/nrg2290.

6. Zheng L., Zhang Y., Liu Y., Zhou M., Lu Y., Yuan L., Zhang C., Hong M., Wang S., Li X. MiR-106b induces cell radioresistance via the PTEN/PI3K/AKT pathways and p21 in colorectal cancer. J Trans/ Med 2015; 13: 252, https://doi. org/10.1186/s12967-015-0592-z.

7. Ma R., Jiang T., Kang X. Circulating microRNAs in cancer: origin, function and application. J Exp Clin Cancer Res 2012; 31: 38-47, https://doi.org/10.1186/1756-9966-31-38.

8. Suzuki H., Maruyama R., Yamamoto E., Kai M. Epigenetic alteration and microRNA dysregulation in cancer. Front Genet 2013; 4: 258, https://doi.org/10.3389/ fgene.2013.00258.

9. Drakaki A., Iliopoulos D. MicroRNA-gene signaling pathways in pancreatic cancer. Biomed J 2013; 36(5): 200208, https://doi.org/10.4103/2319-4170.119690.

10. Metheetrairut C., Slack F.J. MicroRNAs in the ionizing radiation response and in radiotherapy. Curr Opin Genet Dev 2013; 23(1): 12-19, https://doi.org/10.1016/j.gde.2013.01.002.

11. Li G., Qiu Y., Su Z., Ren S., Liu C., Tian Y., Liu Y. Genome-wide analyses of radioresistance-associated miRNA expression profile in nasopharyngeal carcinoma using next generation deep sequencing. PLoS One 2013; 8(12): e84486, https://doi.org/10.1371/journal.pone.0084486.

12. Zhang B., Chen J., Ren Z., Chen Y., Li J., Miao X., Song Y., Zhao T., Li Y., Shi Y., Ren D., Liu J. A specific miRNA signature promotes radioresistance of human cervical cancer cells. Cancer Cell Int 2013; 13(1): 118, https://doi. org/10.1186/1475-2867-13-118.

13. Su H., Jin X., Zhang X., Xue S., Deng X., Shen L., Fang Y., Xie C. Identification of microRNAs involved in the radioresistance of esophageal cancer cells. Cell Biology Int 2014; 38(3): 318-325, https://doi.org/10.1002/cbin.10202.

14. Qu C., Liang Z., Huang J., Zhao R., Su C., Wang S., Wang X., Zhang R., Lee M.H., Yang H. MiR-205 determines the radioresistance of human nasopharyngeal carcinoma by directly targeting PTEN. Cell Cycle 2012; 11(4): 785-796, https://doi.org/10.4161/cc.11.4.19228.

15. Mascotti K., McCullough J., Burger S.R. HPC viability measurement: trypan blue versus acridine orange and propidium iodide. Transfusion 2000; 40(6): 693-696, https:// doi.org/10.1046/j.1537-2995.2000.40060693.x.

16. Müller S., Rycak L., Winter P., Kahl G., Koch I., Rotter B. omiRas: a Web server for differential expression analysis of miRNAs derived from small RNA-Seq data. Bioinformatics 2013; 29(20): 2651-2652, https://doi. org/10.1093/bioinformatics/btt457.

17. Wang J., Le T., Wei R., Jiao Y. Knockdown of JMJD1C, a target gene of hsa-miR-590-3p, inhibits mitochondrial dysfunction and oxidative stress in MPP+-treated MES23.5 and SH-SY5Y cells. Cell Mol Biol 2016; 62(3): 39-45.

18. Villa C., Fenoglio C., De Riz M., Clerici F., Marcone A., Benussi L., Ghidoni R., Gallone S., Cortini F., Serpente M., Cantoni C., Fumagalli G., Martinelli Boneschi F., Cappa S., Binetti G., Franceschi M., Rainero I., Giordana M.T., Mariani C., Bresolin N., Scarpini E., Galimberti D. Role of hnRNP-A1 and miR-590-3p in neuronal death: genetics and expression analysis in patients with Alzheimer disease and frontotemporal lobar degeneration. Rejuvenation Res 2011; 14(3): 275-281, https://doi.org/10.1089/rej.2010.1123.

19. Vaishnavi V., Manikandan M., Tiwary B.K., Munirajan A.K. Insights on the functional impact of microRNAs present in autism-associated copy number variants. PLoS One 2013; 8(2): e56781, https://doi.org/10.1371/journal.pone.0056781.

20. Spencer P., Fry R.C., Kisby G.E. Unraveling 50-year-old clues linking neurodegeneration and cancer to cycad toxins: are microRNAs common mediators? Front Genet 2012; 3: 192, https://doi.org/10.3389/fgene.2012.00192.

21. Chen M., Zhu N., Liu X., Laurent B., Tang Z., Eng R., Shi Y., Armstrong S.A., Roeder R.G. JMJD1C is required for the survival of acute myeloid leukemia by functioning as a coactivator for key transcription factors. Genes Dev 2015; 29(20): 2123-2139, https://doi.org/10.1101/gad.267278.115.

22. Zhu N., Chen M., Eng R., DeJong J., Sinha A.U., Rahnamay N.F., Koche R., Al-Shahrour F., Minehart J.C., Chen C.W., Deshpande A.J., Xu H., Chu S.H., Ebert B.L., Roeder R.G., Armstrong S.A. MLL-AF9- and HOXA9-mediated acute myeloid leukemia stem cell self-renewal requires JMJD1C. J Clin Invest 2016; 126(3): 997-1011, https://doi. org/10.1172/jci82978. 
23. Desgrosellier J.S., Cheresh D.A. Integrins in cancer: biological implications and therapeutic opportunities. Nat Rev Cancer 2010; 10(1): 9-22, https://doi.org/10.1038/ nrc2748.

24. Cordes N., Seidler J., Durzok R., Geinitz H., Brakebusch C. $\beta 1$-integrin-mediated signaling essentially contributes to cell survival after radiation-induced genotoxic injury. Oncogene 2006; 25(9): 1378-1390, https://doi. org/10.1038/sj.onc.1209164.

25. Eke I., Deuse Y., Hehlgans S., Gurtner K., Krause M., Baumann M., Shevchenko A., Sandfort V., Cordes N. $\beta 1$
integrin/FAK/cortactin signaling is essential for human head and neck cancer resistance to radiotherapy. J Clin Invest 2012; 122(4): 1529-1540, https://doi.org/10.1172/jci61350.

26. Bywater M.J., Poortinga G., Sanij E., Hein N., Peck A., Cullinane C., Wall M., Cluse L., Drygin D., Anderes K., Huser N., Proffitt C., Bliesath J., Haddach M., Schwaebe M.K., Ryckman D.M., Rice W.G., Schmitt C., Lowe S.W., Johnstone R.W., Pearson R.B., McArthur G.A., Hannan R.D. Inhibition of RNA polymerase I as a therapeutic strategy to promote cancer-specific activation of p53. Cancer Cell 2012; 22(1): 51-65, https://doi.org/10.1016/j.ccr.2012.05.019. 